\title{
ReaR

\section{Accidente cerebrovascular isquémico y cirugía electiva ¿Cuánto tiempo hay que esperar para una cirugía programada?}

Benito Naverac $H$.

Hospital Clínico Universitario Lozano Blesa de Zaragoza.

\section{Resumen}

En numerosas ocasiones, un paciente que ha sufrido recientemente un ictus, necesita ser intervenido quirúrgicamente de un proceso no neurológico, y se nos plantea la siguiente pregunta.

¿Cuánto tiempo ha de pasar hasta que pueda programarse?

\section{Introducción}

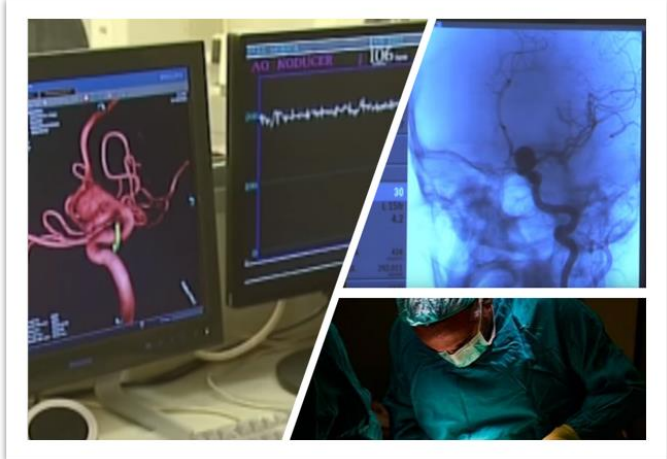

En numerosas ocasiones, un paciente que ha sufrido recientemente un ictus, necesita ser intervenido quirúrgicamente de un proceso no neurológico, y se nos plantea la siguiente pregunta: ¿Cuánto tiempo ha de pasar hasta que pueda programarse?

El accidente cerebrovascular (ACV) se define como la aparición súbita de un déficit neurológico debido a una disrupción focal de la circulación cerebral. El $88 \%$ son isquémicos y el $12 \%$ hemorrágicos.
Es la segunda causa de muerte en el mundo y de discapacidad en Europa. Su prevalencia está aumentando por el envejecimiento de la población. Por otro lado, el ACV perioperatorio es una complicación muy grave que tiene una incidencia en torno al $0,1-0,6 \%$ en cirugía no cardiaca. La etiología parece ser tromboembólica y estar relacionada por el desarrollo de un estado de hipercoagulabilidad postquirúrgico (Tabla 1).

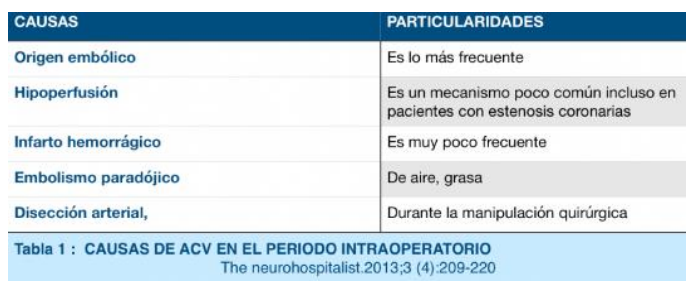

Las intervenciones con más riesgo son las relacionadas con la cirugía cardiaca o la endarterectomía carotídea, tal y como se muestra en la Tabla 2. 


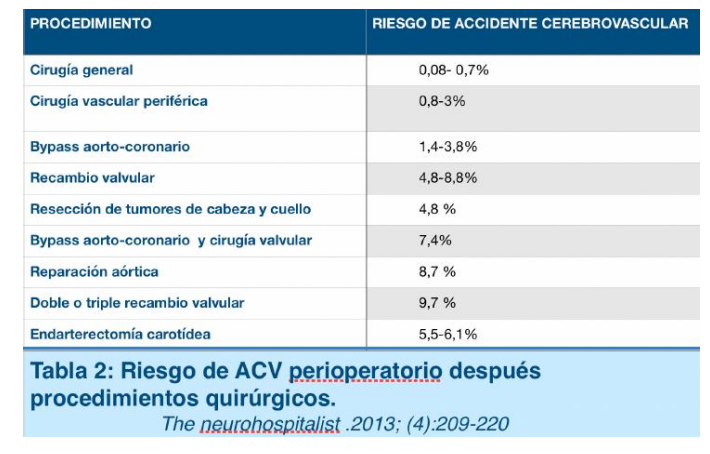

El intervalo de tiempo ideal que tiene que transcurrir después de un accidente cerebrovascular para programar con seguridad una cirugía electiva, no ha sido determinado de forma científica. La mayor parte de los trabajos son observacionales y sus resultados variables según el estudio que consultemos. La variabilidad de la enfermedad cerebrovascular en cuanto a su severidad, signos $\mathrm{y}$ síntomas, y territorios cerebrales afectados y la variabilidad de indicaciones quirúrgicas que se nos pueden presentar hace muy difícil el obtener conclusiones válidas.

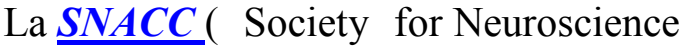
un Anesthesiology and Critical Care) recomienda esperar un intervalo de tiempo de 1 a 3 meses. Según los datos de Jørgensen et al, se propone esperar al menos 6 meses después del ACV. En las guías de la SEDAR de recomendaciones de manejo perioperatorio de antiagregantes plaquetarios en cirugía no cardiaca se afirma que:" el antecedente de ictus 9 meses antes de la intervención aumenta el riesgo no sólo de recurrencia de ictus, sino también se asocia a un aumento de las complicaciones cardiovasculares y de mortalidad"

\section{Las principales razones para esperar son las siguientes:}

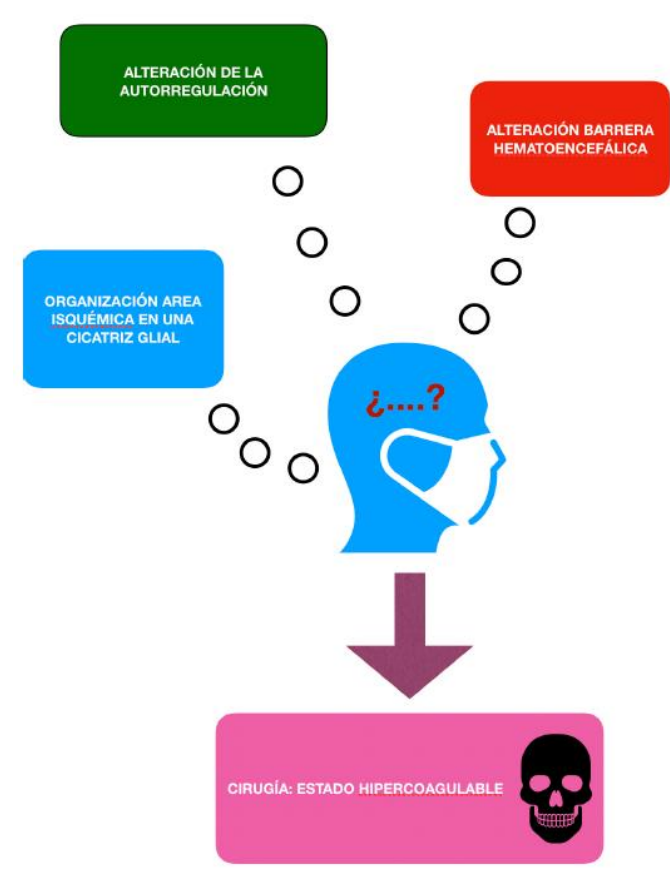

1. Tras sufrir un ACV la autorregulación permanece deteriorada durante más de tres meses. Esto hace que el flujo sanguíneo cerebral sea dependiente de los niveles de presión arterial, por lo que aumenta el riesgo de sufrir hipoperfusión cerebral si son sometidos a una cirugía con sangrado e hipotensión.

2. La barrera hematoencefálica se encuentra alterada por fenómenos inflamatorios al menos durante 4-6 semanas.

3. La organización del área infartada en una cicatriz glial lleva algo de más tiempo.

4. Para realizar algunas cirugías hay que interrumpir el tratamiento antiagregante lo cual favorece la reaparición del evento cerebrovascular.

5. El estado de los pacientes que sufren un deterioro funcional severo puede empeorar si se les somete a una cirugía electiva temprana.

Guía de actuación en caso de que la cirugía no pueda demorarse 
1. No siempre es posible demorar una intervención quirúrgica, (por ejemplo en una cirugía oncológica o en una fractura de cadera), en estos casos hay que hacer una evaluación preoperatoria exhaustiva $\mathrm{y}$ valorar la aplicación de las siguientes medidas:

2. Tener en cuenta el riesgo hemorrágico. Si el riesgo de sangrado no es muy elevado valorar intervenir sin suspender AAS $100 \mathrm{mg}$ o suspendiéndolo 24 horas antes.

3. En cuanto al manejo de otros antiagregantes se recomienda suspender el clopidogrel al menos 5-7 días antes de la cirugía. Sustituir adiro $300 \mathrm{mg}$ por adiro $100 \mathrm{mg} 7$ días previos a la cirugía.

4. El tratamiento con estatinas puede ayudar a prevenir la recurrencia del ictus por varios mecanismos, ya que poseen acción antiinflamatoria. por lo que es aconsejable continuar con el tratamiento durante el periodo perioperatorio. La retirada de estos fármacos eleva casi por 8 el riesgo de recurrencia, asociándose a una mayor morbimortalidad.

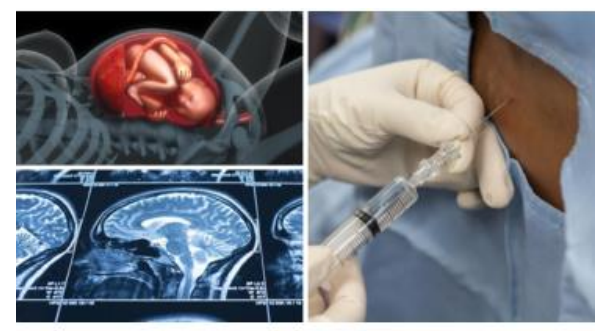

19 febrero 2018

\section{Anestesia neuroaxial en gestantes con patología intracraneal}

5. Optar por anestesia locorregional, siempre que sea posible. La anestesia raquídea puede ser una opción siempre que no haya una lesión ocupante de espacio que cause hipertensión intracraneal (riesgo de enclavamiento).

6. Si es necesario hacer una anestesia general, la intubación orotraqueal debe realizarse de forma suave y cuidadosa. Evitar la hipoxia y la hipercapnia. Ningún fármaco ha demostrado superioridad en este contexto. Es prioritario mantener la estabilidad hemodinámica.

7. Conseguir unas cifras de presión de perfusión cerebral ( $\mathrm{PPC}=$ PAM-PIC) adecuadas. Para ello se recomienda que las cifras de PAM se encuentren entre 70-100 mmHg. Es especialmente importante evitar la aparición de hipotensión arterial, ya que la autorregulación del flujo sanguíneo cerebral se encuentra inhibida $y$ es fácil que se produzca hipoperfusión cerebral.

8. El uso de oximetría cerebral no invasiva ,podría ayudarnos a detectar episodios de desaturación cerebral.

9. Evitar la hipertermia (temperatura $>38^{\circ} \mathrm{C}$ ). Tratar la fiebre con antipiréticos si es necesario. La hipotermia aumenta el consumo cerebral de oxígeno por lo que no se recomienda que la del paciente no baje por debajo de $36^{\circ} \mathrm{C}$.

10. Control estricto de la glucemia sanguínea. Tratar la hipoglucemia $\quad(<60 \quad \mathrm{mg} \quad /$ dl). Mantener las cifras de glucemia entre $140-180 \mathrm{mg} / \mathrm{dl}$.

11. La barrera hematoencefálica se encuentra alterada, por lo que es

12. necesaria la realización de un control estricto del tipo y la cantidad de fluidos que se le administran al paciente. El 
objetivo es conseguir que el paciente esté normovolémico. Evitar los líquidos hipotónicos respecto del plasma tales como el Ringer Lactato ${ }^{\circledR}$. De elección es el suero fisiológico.

\section{Valoración preoperatoria del paciente con $\mathrm{ACV}$ isquémico reciente y pendiente de cirugía}

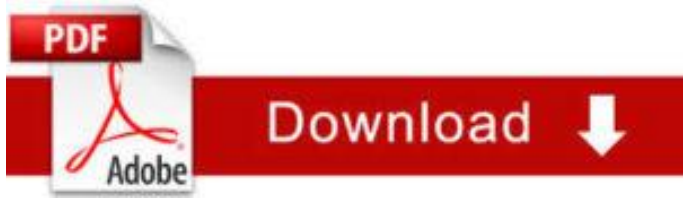

\section{Conclusiones}

A pesar de la poca evidencia científica existente al respecto, parece razonable esperar entre 6-9 meses a realizar la cirugía electiva en los pacientes que han sufrido un accidente cerebrovascular. En caso de que la intervención no pueda demorarse, se recomienda NO interrumpir el tratamiento con estatinas durante el periodo perioperatorio y valorar cuidadosamente el balance "riesgo hemorrágico/ riesgo trombótico" a la hora de suspender el tratamiento antiagregante plaquetario.

\section{Bibliografía}

1. Wong GY, Warner DO, Schroeder DR, Offord KP, Warner MA, Maxson PM, Whisnant JP. Risk of surgery and anesthesia for ischemic stroke. Anesthesiology. 2000;92(2) : :425432 (HTML)

2. Hudson KA, Greene JG. Perioperative consultation for patientes with preexisting neurologic disorders. Semin Neurol.2015; 35(6): 6908. (Pubmed)

3. Sanders R.D, Jørgensen ME, Mashour G.A. Perioperative stroke: a question of timing? BJA 2015; 115(1):1113 (HTML)

4. Probasco J, Sahin B, Tran T, Chung TH, Rosenthal LS, Mari Z, Levy M. The preoperative neurological evaluation Neurohospitalist. 2013;3(4):209-220. (HTML)

5. Vlisides $\mathrm{P}$, Mashour $\mathrm{G}$ A. Perioperative Stroke. Can J Anesthesia 2016; 63(2): 193-204(HTML)

6. Jauch EC, Saver JL, Adams Jr HP, et al. Guidelines for the early management of patients with acute ischemic stroke: a guideline for healthcare professionals from the American Heart Association and American Stroke Association. Stroke. 2013;44:870-947. (HTML)

7. 2018 Guidelines for the Early Management of Patients with Acute Ischemic Stroke. Stroke.2018;49:e46e99. (HTML)

8. Ghosh A, Elwell C, Martin S. Cerebral Near- infrared Spectroscopy in adults. A work in progress. Anesthesia\&Analgesia 2012; 115(6):1373-1383 (PubMed)

9. Christiansen MN, Anderson C, Gunnar $\mathrm{H}$, Torp-Pedersen $\mathrm{C}$ et al. Risks of cardiovascular adverse events and death in patients with previous stroke undergoing emergency non cardiac, nonintracranial surgery. The importance of operative timing. Anesthesiology 2017; 127 : 919. (PubMed)

10. Bagre AS. Perioperative Stroke. Prediction, Prevention and protection. Indian Journal of Anesthesia 2018; 62(0): 738-742.(HTML)

11. Mashour G, Moore $\mathrm{L}$ et al. Perioperative Care of pacientes at high risk for stroke during or after NonCardiac, Non - Neurologic Surgery: Consensus Statement from the Society for

Neuroscience in Anesthesiology and Critical Care. J Neurosug Anesthesiol. 2014; 26(4):273-285 (PubMed)

Correspondencia al autor

Helena Benito Naverac

helenabenito@yahoo.es

FEA de Anestesiología y Reanimación.

Hospital Clínico Universitario Lozano Blesa de Zaragoza.

Aceptado para el blog en febrero de 2019. 